Internat. J. Math. \& Math. Sci.

Vol. 24, No. 1 (2000) 43-48

S0161171200003306

(C) Hindawi Publishing Corp.

\title{
FIRST EIGENVALUE OF SUBMANIFOLDS IN EUCLIDEAN SPACE
}

\author{
KAIREN CAI
}

(Received 23 April 1999)

\begin{abstract}
We give some estimates of the first eigenvalue of the Laplacian for compact and non-compact submanifold immersed in the Euclidean space by using the square length of the second fundamental form of the submanifold merely. Then some spherical theorems and a nonimmersibility theorem of Chern and Kuiper type can be obtained.
\end{abstract}

Keywords and phrases. Laplacian, eigenvalue, submanifold.

2000 Mathematics Subject Classification. Primary 53C42.

1. Introduction. Let $M$ be an $n$-dimensional compact connected submanifold immersed in the Euclidean space $\mathbb{R}^{n+p}$. Denote by $\|\sigma\|^{2}$ and $\lambda_{1}$ the square length of the second fundamental form and the first eigenvalue of the Laplacian of $M$. It is well known that if $M$ is a standard hypersphere in the Euclidean space $\mathbb{R}^{n+1}$, then $\lambda_{1}=n$. We find that $\|\sigma\|^{2}$ is equal to $n$ at the same time, i.e., $\lambda_{1}=\|\sigma\|^{2}$. Inspiring the exterior rigidity of sphere, a natural problem appears: can you characterize those submanifolds immersed in $\mathbb{R}^{n+p}$ as $n$-sphere by $\lambda_{1}$ and $\|\sigma\|^{2}$ ?

The main goal of this paper is to give an affirmative answer for this question. In fact we can prove the following further result.

THEOREM 1.1. Let $M$ be a compact submanifold immersed in the Euclidean space $\mathbb{R}^{n+p}$. Denote by $\|\sigma\|^{2}$ the square length of the second fundamental form and $\lambda_{1}$ the first eigenvalue of the Laplacian of $M$. Then $\lambda_{1} \leq \max _{M}\|\sigma\|^{2}$. Furthermore, if $\lambda_{1} \geq\|\sigma\|^{2}$ holds at any point of $M$, then $M$ is isometric to a sphere $S^{n}$.

According to Nash's imbedded theorem, every Riemannian manifold can be isometrically imbedded in a Euclidean space of sufficiently large dimension. It is very significant to investigate the geometry of submanifold of the Euclidean space. For example, in the case of an $n$-dimensional compact hypersurface immersed in the sphere $S^{n+1}(c)$ with constant curvature $c$ in the Euclidean space $\mathbb{R}^{n+2}$, similar conclusion can be obtained immediately as follows.

THEOREM 1.2. Let $M$ be a compact hypersurface immersed in the sphere $S^{n+1}(c)$. Denote by $\|\sigma\|^{2}$ the square length of the second fundamental form and $\lambda_{1}$ the first eigenvalue of the Laplacian of $M$. Then $\lambda_{1} \leq n c+\max _{M}\|\sigma\|^{2}$. Furthermore, if $\lambda_{1} \geq$ $n c+\|\sigma\|^{2}$ holds at any point of $M$, then $\|\sigma\|^{2}=0$ and $M$ is isometric to a totally geodesic sphere $S^{n}(c)$.

In fact we set up a sharp estimate of the upper bound for the first eigenvalue of $M$ in $\mathbb{R}^{n+p}$ by using merely $\|\sigma\|^{2}$. A useful version of the lower bound for the Ricci 
curvature of submanifold stated as a lemma will be given. The lemma can be applied not only to the estimate of the first eigenvalue for both compact and non-compact submanifolds in the Euclidean space $\mathbb{R}^{n+p}$, but in some propositions of the geometry of submanifolds (see [2, 7]). As is well known, this type of theorems of compact hypersurfaces in the Euclidean space $\mathbb{R}^{n+1}$ was also proven by some authors such as Reilly, Ros, and Deshmukh (see [4, 8, 9]). Deshmukh obtained similar results under the condition that $M$ is a strictly convex hypersurface immersed in $\mathbb{R}^{n+1}$. We shall deal with the more general case without the assumption of convexity of hypersurfaces.

As an application in the proof of Theorem 1.1, a new nonimmersibility theorem of Chern and Kuiper type [3] can be obtained as follows.

THEOREM 1.3. Let $M$ be an $n$-dimensional compact Riemannian manifold whose Ricci curvature Ric and scalar curvature $R$ satisfy $\operatorname{Ric}(v, v)+R \geq 0$ and $R<n(n-1) \lambda^{-2}$ for each unit vector field $v$ and some constant $\lambda>0$. Then no isometric immersion of $M$ into the Euclidean space $\mathbb{R}^{n+1}$ is contained in a ball $B^{n+1}$ of radius $\lambda$.

Deshmukh and Al-Gwaiz [5] proved a similar result under the assumption that the dimension of manifolds should be odd. Furthermore, when the dimension of $M$ is odd say $2 m-1$, the condition $R<2(2 m-1)(m-1) \lambda^{-2}$ in Theorem 1.3 is better than Ric $<2(m-1) \lambda^{-2}$ stated in [5].

2. Preliminaries. Let $M$ be a compact submanifold immersed in $\mathbb{R}^{n+p}$. Take a local orthonormal frame field $\left\{e_{1}, \ldots, e_{n+p}\right\}$ in $\mathbb{R}^{n+p}$ around a point $p \in M$ such that when restricting on $M,\left\{e_{1}, \ldots, e_{n}\right\}$ are tangent to $M$ and $\left\{e_{n+1}, \ldots, e_{n+p}\right\}$ are normal to $M$. Let $\nabla, \bar{\nabla}$, and $\bar{\nabla}^{\perp}$ be the Riemannian connections on $\mathbb{R}^{n+p}, T M$, and $(T M)^{\perp}$, respectively. The Gauss and Weingarten formulas are

$$
\bar{\nabla}_{X} Y=\nabla_{X} Y+B(X, Y), \quad \bar{\nabla}_{X} e_{\alpha}=-A_{\alpha} X+\bar{\nabla}_{X}^{\frac{1}{X}} e_{\alpha},
$$

where $n+1 \leq \alpha \leq n+p, X, Y$ are vector fields on $M$. Denote $H_{\alpha}$ the trace of the Weingarten transformation $A_{\alpha}$, then the mean curvature of the immersion can be written as

$$
H=\frac{1}{n} \sqrt{\sum_{\alpha} H_{\alpha}^{2}}
$$

From the Gauss equation we have

$$
\begin{gathered}
\operatorname{Ric}(X, Y)=H_{\alpha}\left\langle A_{\alpha}(X), Y\right\rangle-\left\langle A_{\alpha}(X), A_{\alpha}(Y)\right\rangle, \\
R=n^{2} H^{2}-\|\sigma\|^{2},
\end{gathered}
$$

where Ric and $R$ are the Ricci curvature and the scalar curvature of $M$. We accept the convention that the double indexes mean the summation.

Let $x: M \rightarrow \mathbb{R}^{n+p}$ be an isometric immersion. For $q \in M, x(q)$ also means the position vector of $q$ with origin zero. The support function $\rho_{\alpha}: M \rightarrow R$ of the immersion $x$ is given by

$$
\rho_{\alpha}(q)=\left\langle x, e_{\alpha}\right\rangle_{q}
$$


We define $M \rightarrow R$ by $f=(1 / 2)\|x\|^{2}$ as Reilly did in [8]. Let us denote by $\nabla f$ the gradient of the function $f$. Then

$$
x=\nabla f+\rho_{\alpha} e_{\alpha}
$$

Proof OF TheOrem 1.1. From the definition of the Riemannian curvature operator

$$
R(X, Y) Z=\left(\nabla_{X} \nabla_{Y} Z-\nabla_{Y} \nabla_{X} Z-\nabla_{[X, Y]} Z\right),
$$

we get

$$
R\left(e_{i}, e_{j}\right) \nabla f=\left(\nabla_{e_{i}} \nabla_{e_{j}}-\nabla_{e_{j}} \nabla_{e_{i}}\right) \nabla f .
$$

Without loss of generality we suppose $\left.\nabla_{e_{i}} e_{j}\right|_{q}=0$ for $q \in M$. Hence

$$
\operatorname{Ric}(\nabla f, \nabla f)=\left\langle\left(\nabla_{e_{i}} \nabla_{e_{j}}-\nabla_{e_{j}} \nabla_{e_{i}}\right) \nabla f, e_{i}\right\rangle\left\langle\nabla f, e_{j}\right\rangle .
$$

Integrating both sides of (2.9) and using the divergence theorem, it follows that

$$
\int_{M}\left\langle\nabla_{e_{i}} \nabla f, e_{i}\right\rangle^{2}-\|\nabla \nabla f\|^{2}-\operatorname{Ric}(\nabla f, \nabla f)=0 .
$$

We have at $q$,

$$
\nabla_{X} \nabla f=\left\langle\bar{\nabla}_{X} x, e_{j}\right\rangle e_{j}+\left\langle x, \bar{\nabla}_{X} e_{j}\right\rangle e_{j}=X+\left\langle x, B\left(X, e_{j}\right)\right\rangle e_{j}=X+\rho_{\alpha} A_{\alpha}(X) .
$$

Hence

$$
\Delta f=n+\rho_{\alpha} H_{\alpha} .
$$

Integrating both sides of (2.12) and using Stokes theorem, we get

$$
\int_{M} n+\rho_{\alpha} H_{\alpha}=0
$$

When $p=1$ the expression becomes the classical Minkowski formula. It follows from (2.11) that

$$
\left\langle\nabla e_{i} \nabla f, e_{i}\right\rangle^{2}=n^{2}+2 n \rho_{\alpha} H_{\alpha}+\left(\rho_{\alpha} H_{\alpha}\right)^{2}, \quad\|\nabla \nabla f\|^{2}=n+2 \rho_{\alpha} H_{\alpha}+\left\|\rho_{\alpha} A_{\alpha}\right\|^{2} .
$$

Substituting (2.14) in (2.10), we reach

$$
\int_{M} \rho_{\alpha} \rho_{\beta}\left(H_{\alpha} H_{\beta}-\left\langle A_{\alpha} A_{\beta}\right\rangle\right)-\operatorname{Ric}(\nabla f, \nabla f)=n(n-1) \operatorname{Vol} M,
$$

where $\operatorname{Vol} M$ expresses the volume of $M$. We take the center of mass of $M$ as the origin zero of $\mathbb{R}^{n+p}$. Then $\int_{M} x=0$. According to the max-minimum principle we get

$$
n \operatorname{Vol} M=-\int_{M}\langle\Delta x, x\rangle \geq \lambda_{1} \int_{M}\|x\|^{2},
$$


in which $\lambda_{1}$ is the first eigenvalue of $M$. Using an orthogonal transformation to $\left\{e_{n+1}\right.$, $\left.\ldots, e_{n+p}\right\}$, we can make the symmetric matrix $\left(H_{\alpha} H_{\beta}-\left\langle A_{\alpha}, A_{\beta}\right\rangle\right)$ to be diagonal at $q \in M$. Without loss of generality, we may assume that $\left(H_{\alpha} H_{\beta}-\left\langle A_{\alpha}, A_{\beta}\right\rangle\right)$ is diagonal at $q$. By using the Schwartz inequality it follows that

$$
\sum_{\alpha} \rho_{\alpha}^{2}\left(H_{\alpha}^{2}-\left\|A_{\alpha}\right\|^{2}\right) \leq(n-1) \sum_{\alpha} \rho_{\alpha}^{2}\left\|A_{\alpha}\right\|^{2} \leq(n-1)\|\sigma\|^{2} \sum_{\alpha} \rho_{\alpha}^{2} .
$$

We get from (2.15), (2.16), and (2.17)

$$
\lambda_{1}\|x\|^{2} \leq \int_{M}\|\sigma\|^{2} \sum_{\alpha} \rho_{\alpha}^{2}-\frac{1}{n-1} \operatorname{Ric}(\nabla f, \nabla f) .
$$

It follows from the following lemma that

$$
\operatorname{Ric}(\nabla f, \nabla f) \geq-\frac{\sqrt{n-1}}{2}\|\sigma\|^{2}\|\nabla f\|^{2} .
$$

Therefore,

$$
\lambda_{1} \int_{M}\|x\|^{2} \leq \int_{M}\|\sigma\|^{2}\left(\sum_{\alpha} \rho_{\alpha}^{2}+\frac{1}{2 \sqrt{n-1}}\|\nabla f\|^{2}\right) .
$$

Then we reach

$$
\lambda_{1} \leq \max _{M}\|\sigma\|^{2}
$$

If the equality in (2.21) holds, then the equalities in (2.17), (2.19), and (2.20) also appear. Hence $\nabla f=0,\|\sigma\|^{2}=$ constant and $M$ lies in a sphere $S^{n+p-1}$. From (2.17) we get $\sum_{\alpha} \rho_{\alpha}^{2}\left\|A_{\alpha}\right\|^{2}=\|\sigma\|^{2} \sum_{\alpha} \rho_{\alpha}^{2}$, so it concludes that for some $\alpha$, say $\alpha=n+1,\left\|A_{n+1}\right\|^{2}=$ $\|\sigma\|^{2}$ and $\left\|A_{n+2}\right\|^{2}=\cdots=\left\|A_{n+p}\right\|^{2}=0$. Then $M$ lies in a totally geodesic $S^{n+1}$. As $M$ is isometrically a closed submanifold in the Euclidean sphere, $M$ should be isometric to a sphere in $\mathbb{R}^{n+1}$ with radius $r=n /\|\sigma\|^{2}$. This ends the proof of Theorem 1.1.

REMARK 2.1. It is an interesting fact that one can find the upper bounds of the first eigenvalue for some kind of hypersurfaces by using Theorem 1.1. For example, as well known, the Clifford hypersurfaces $M_{p} \times M_{q}=S^{p}\left(1 /\left(\sqrt{1+\lambda^{2}}\right)\right) \times S^{q}\left(\lambda /\left(\sqrt{1+\lambda^{2}}\right)\right)$, where integers $p+q=n$, are compact hypersurfaces in $S^{n+1}$ with constant $\|\sigma\|^{2}=$ $n+p \lambda^{2}+q / \lambda^{2}$ (see [3]), then we have $\lambda_{1}\left(M_{p} \times M_{q}\right) \leq n+p \lambda^{2}+q / \lambda^{2}$.

3. Lemma and corollaries results. We need the following lemma.

LEMMA 3.1. Let $M$ be an n-dimensional submanifold immersed in a Riemannian manifold $N^{n+p}$. Denote by Ric and $\left\|\sigma_{N}\right\|^{2}$ the functions on $M$ that assign to each point of $M$ the minimum Ricci curvature and the square length of the second fundamental form at the point, respectively. If all the sectional curvatures of $N^{n+p}$ are bounded below by $k$, then

$$
\text { Ric } \geq(n-1) k-\frac{\sqrt{n-1}}{2}\left\|\sigma_{N}\right\|^{2}
$$


Proof. It is known from Cai and Leung (see $[2,7])$ that

$$
\text { Ric } \geq \frac{n-1}{n}\left\{n k+n H^{2}-\|\varphi\|^{2}-\frac{n-2}{\sqrt{n-1}} \sqrt{n H^{2}}\|\varphi\|\right\},
$$

where $H$ is the mean curvature of the immersion and $\|\varphi\|^{2}=\left\|\sigma_{N}\right\|^{2}-n H^{2}$ (see [1]). Let us consider the quadratic form with eigenvalues $\pm n / 2 \sqrt{n-1}$ :

$$
F(x, y)=x^{2}-\frac{n}{2 \sqrt{n-1}} x y-y^{2} .
$$

By using an orthogonal transformation, $F(x, y)$ can be written as

$$
F(x, y)=\frac{n}{2 \sqrt{n-1}}\left(u^{2}-v^{2}\right) .
$$

Let $x=\sqrt{n H^{2}}, y=\|\varphi\|$ then $x^{2}+y^{2}=\left\|\sigma_{N}\right\|^{2}$. It follows from $x^{2}+y^{2}=u^{2}+v^{2}$ that

$$
\text { Ric } \geq(n-1) k+\frac{\sqrt{n-1}}{2}\left(u^{2}-V^{2}\right) \geq(n-1) k-\frac{\sqrt{n-1}}{2}\left\|\sigma_{N}\right\|^{2} \text {. }
$$

Thus we derived the conclusion.

In the case of complete non-compact submanifolds in $\mathbb{R}^{n+p}$, Gage (see [6]) proved that $\lambda_{1} \leq-(n-1) / 4$ Ric. Together with Lemma 3.1, we obtain the following corollary.

COROLlary 3.2. Let $M$ be an n-dimensional complete non-compact submanifold immersed in $\mathbb{R}^{n+p}$. Then

$$
\lambda_{1}(M) \leq \frac{n-1}{8} \sqrt{n-1} \sup _{M}\|\sigma\|^{2} .
$$

Now we consider the case of $p=1$ in which $M$ is a closed hypersurface immersed in $\mathbb{R}^{n+1}$. By using Lemma 3.1 and (2.4) in (2.15) we get

$$
(n-1) \lambda_{1} \leq \int_{M} \frac{\left(R \rho^{2}+(\sqrt{n-1} / 2)\|\sigma\|^{2}\|\nabla f\|^{2}\right)}{\left(\int_{M} \rho^{2}+\|\nabla f\|^{2}\right)} \leq \max _{M}\left(R, \frac{\sqrt{n-1}}{2}\|\sigma\|^{2}\right) .
$$

Hence we obtain the following corollary.

COROLLARY 3.3. Let $M$ be a closed hypersurface immersed in $\mathbb{R}^{n+1}$. Then

$$
\lambda_{1} \leq \max _{M}\left\{\frac{R}{n-1}, \frac{1}{2 \sqrt{n-1}}\|\sigma\|^{2}\right\}
$$

and the equality holds if and only if $M$ is isometric to a sphere $S^{n}(r)$ with radius $r$.

As is well known, a hypersurface in $\mathbb{R}^{n+1}$ possessing the non-negative Ricci curvature implies that it is a convex hypersurface of $\mathbb{R}^{n+1}$. Thus we can easily get from (2.15) and (2.16) the following.

COROLLARY 3.4. Let $M$ be a closed convex hypersurface immersed in $\mathbb{R}^{n+1}$. If $R \leq$ $(n-1) \lambda_{1}$ holds for all points of $M$. Then $M$ is isometric to a sphere $S^{n}(r)$.

REMARK 3.5. Here we only need the condition Ric $\geq 0$ rather than Ric $>0$ which was assumed by Deshmukh in [4]. We should point out that [4, Theorem 2] is very obvious by means of the expression $\Delta f=n(1+\rho H)$ and the property of harmonic functions on the compact Riemannian manifold. 
Proof OF TheORem 1.3. Suppose that there exists an isometric immersion $x$ : $M \rightarrow \mathbb{R}^{n+1}$ such that $x(M)$ is contained in a ball $B^{n+1}$ of $\mathbb{R}^{n+1}$ with radius $\lambda$. For $p=1$ from (2.4), (2.15) becomes

$$
\int_{M} \rho^{2} R-\operatorname{Ric}(\nabla f, \nabla f)-n(n-1)=0 .
$$

Now, we observe that the vector field $\nabla f$ is not identically zero on $M$. For if $\nabla f \equiv 0$, then $f=$ constant, say $f=(1 / 2) r^{2}$ on $M$. We conclude that $M$ is a sphere with radius $r$. So $R=n(n-1)\|x\|^{-2}$, it contradicts the hypothesis $R<n(n-1) \lambda^{-2}$. Then we can let $v=\nabla f /\|\nabla f\|$ is the unit position vector field defined on the open subset of $M$ where $\nabla f$ is non-zero. Using $\|x\|^{2}=\|\nabla f\|^{2}+\rho^{2}$ in the integral formula (3.9), we obtain

$$
\int_{M}\|\nabla f\|^{2}(\operatorname{Ric}(v, v)+R)+n(n-1)-\|x\|^{2} R=0 .
$$

From this hypothesis of the theorem it follows that $\operatorname{Ric}(v, v)+S \geq 0$ and $\|x\|^{2} R \leq$ $\lambda^{2} R<n(n-1)$, we obtain a contradiction to (3.10). This ends the proof of Theorem 1.3.

ACKNOWLEDGEMENT. The author is grateful to the School of Mathematics, University of Bristol for their hospitality during his visit in 1998. Projects were supported by the National Natural Science Foundation of China.

\section{REFERENCES}

[1] H. Alencar and M. do Carmo, Hypersurfaces with constant mean curvature in spheres, Proc. Amer. Math. Soc. 120 (1994), no. 4, 1223-1229. MR 94f:53108. Zbl 802.53017.

[2] K. R. Cai, Topology of some closed submanifolds in Euclidean space, Chinese Ann. Math. Ser. A 8 (1987), no. 2, 234-241. MR 89g:53091. Zbl 638.53055.

[3] S. Chern and N. H. Kuiper, Some theorems on the isometric imbedding of compact Riemannian manifolds in euclidean space, Ann. of Math. (2) 56 (1952), 422-430. MR 14,408e. Zbl 049.23402.

[4] S. Deshmukh, Compact hypersurfaces in a euclidean space, Quart. J. Math. Oxford Ser. (2) 49 (1998), no. 193, 35-41. MR 99h:53083. Zbl 906.53003.

[5] S. Deshmukh and M. A. Al-Gwaiz, Compact hypersurfaces in even-dimensional euclidean space and in the sphere, Quart. J. Math. Oxford Ser. (2) 45 (1994), no. 178, 151-157. MR 95d:53060. Zbl 810.53046.

[6] M. E. Gage, Upper bounds for the first eigenvalue of the Laplace-Beltrami operator, Indiana Univ. Math. J. 29 (1980), no. 6, 897-912. MR 82b:58095. Zbl 465.53031.

[7] P. F. Leung, An estimate on the Ricci curvature of a submanifold and some applications, Proc. Amer. Math. Soc. 114 (1992), no. 4, 1051-1061. MR 92g:53052. Zbl 753.53003.

[8] R. C. Reilly, Applications of the Hessian operator in a Riemannian manifold, Indiana Univ. Math. J. 26 (1977), no. 3, 459-472. MR 57\#13799. Zbl 391.53019.

[9] A. Ros, Compact hypersurfaces with constant higher order mean curvatures, Rev. Mat. Iberoamericana 3 (1987), no. 3-4, 447-453. MR 90c:53160. Zbl 673.53003.

Kairen Cai: Department of Mathematics, Hangzhou Teachers' College, 96 Wen Yi ROAD, HANGZHOU 310012, CHINA

E-mail address: kcai@mai 1.hz.zj.cn 


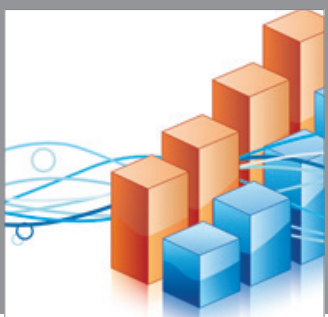

Advances in

Operations Research

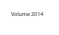

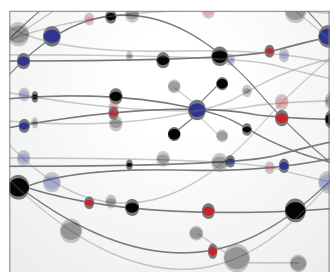

\section{The Scientific} World Journal
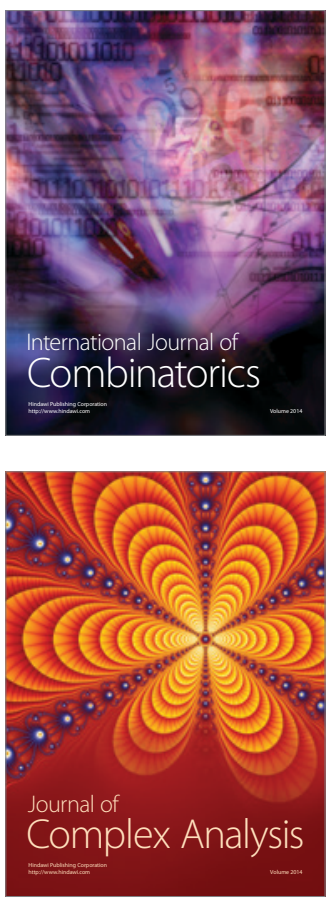

International Journal of

Mathematics and

Mathematical

Sciences
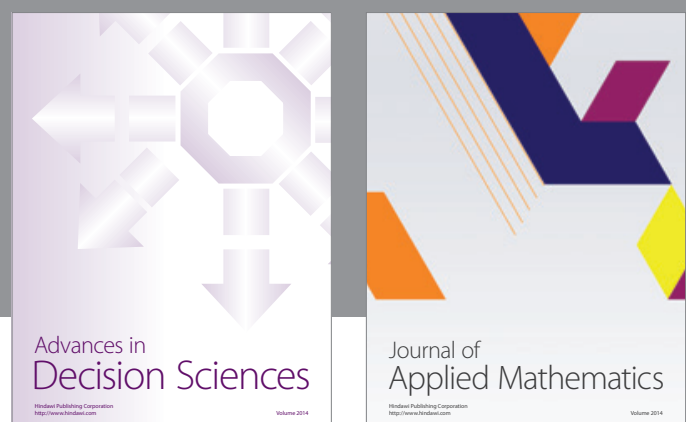

Journal of

Applied Mathematics
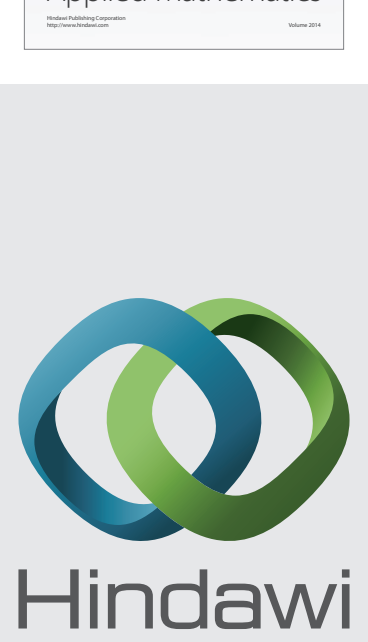

Submit your manuscripts at http://www.hindawi.com
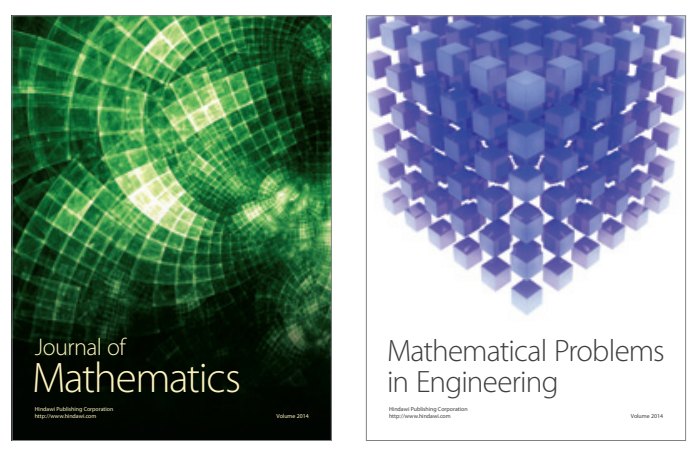

Mathematical Problems in Engineering
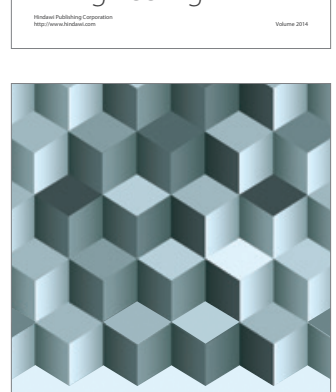

Journal of

Function Spaces
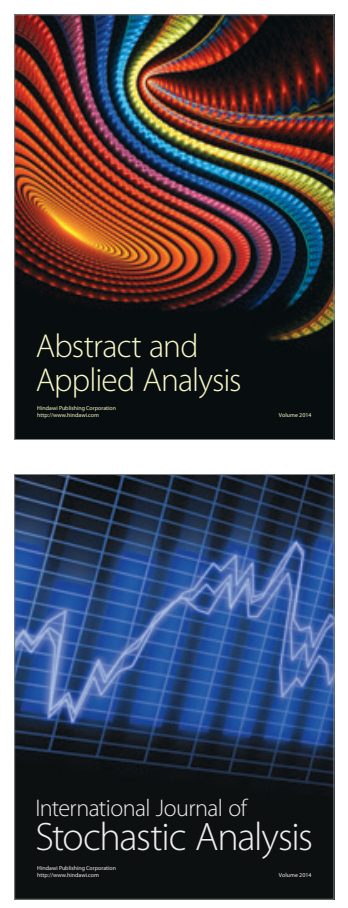

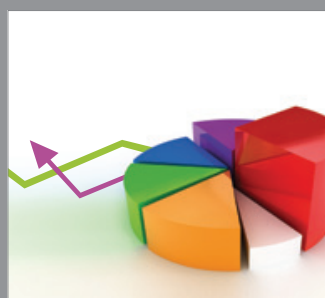

ournal of

Probability and Statistics

Promensencen
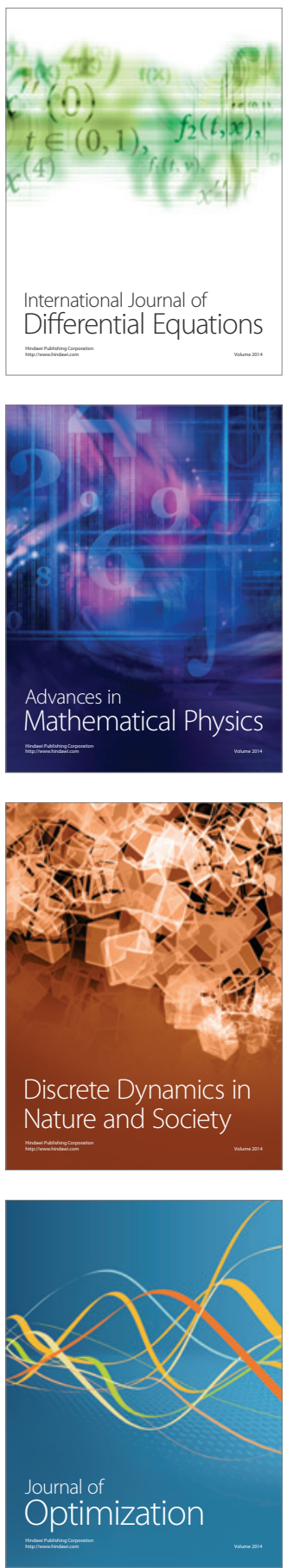